\title{
Research on Reliability Distribution Technology of CNC Lathe HTC2050
}

\author{
Miaomiao Zhang ${ }^{1}$, Yan $\mathrm{Cao}^{2}$, Zhou Fang ${ }^{3}$ and Bei Chen ${ }^{4}$ \\ ${ }^{1}$ Department of Mechanical and Electronic Engineering, Xi'an Technological University, \\ Xi'an, China \\ 654662115@qq.com,jantonyz@163,27381788@qq.com,651723489@qq.com
}

\begin{abstract}
By studying the reliability distribution of CNC lathe, it is possible to solve the problem of poor reliability and short life of $\mathrm{CNC}$ lathe, which can also reveal the weak links, key elements and components in the system design. The mathematical model of NC lathe is established by using Arlins distribution method, and the importance degree of each subsystem in $\mathrm{CNC}$ lathe is analyzed quantitatively based on the reliability allocation criterion. The reliability of all subsystems in the mechanical system and the feasibility of the Arlins distribution method are verified by an example.
\end{abstract}

Keywords: CNC lathe; Reliability distribution; Arlins distribution

\section{Introduction}

Reliability distribution is essential to the reliability design of CNC lathe. The purpose is to make full use of limited resources. The principle of reliability allocation is assigning the reliability index reasonably to the subsystems in the system, which can determine the subsystem or component reliability index in order to ensure the reliability of CNC lathe indicators. The low level of reliability and short service life are two of the biggest obstacles in Chinese machinery industry, which limit the export of our products engineering machinery, especially $\mathrm{CNC}$ lathes. In addition to the poor domestic CNC lathe reliability and low maintenance in the use process of product, more breakdown and the short life directly also affect the production efficiency and the social efficiency, which main reason is paying less attention to the numerical control lathe reliability research in the past, so that the data of failure has not been collected and analyzed ${ }^{[4]}$. Thus, China's CNC lathe reliability still has large differences compared with developed countries. In the reliability distribution of CNC lathe, the common using approach is equal distribution method ${ }^{[1]}$ used in product design early, which is very simple but does not consider the components of the existing estimates (and re-allocation issues) and does not take into account the different properties between the various components, such as importance and complexity are different. In fact, the previous method is a simple distribution of the average. It requires accurate allocation of the reliability index of each component according to the degree of importance in the system, otherwise it cannot be used. The novel method of distribution is to consider the importance of parts by a distribution method and is the basis for product reliability design. The importance of the subsystems is different in the numerical control lathe, and the use of the Arlins distribution method can be important for each subsystem to give a quantitative description of the system, which is of great value to the system design and failure analysis.

\section{CNC lathe reliability allocation criteria}

The reliability distribution of $\mathrm{CNC}$ lathe system is the process of assigning the reliability index of $\mathrm{NC}$ lathe system to each sub-system of CNC lathe so as to get the reliability index requirement of CNC lathe subsystems and to ensure the design and manufacture. Reliability allocation can make the design work clearly and know what level of reliability of each subsystem without blindly design, thus improving design efficiency while reducing design costs. In the reliability distribution of CNC lathe subsystems. We need check to determine whether it meets the requirements through the calculation according to the complexity of each subsystem, the working environment and other aspects of the general reliability allocation.

In the reliability distribution of $\mathrm{CNC}$ lathe subsystems, the following guidelines should be followed.

For highly complex subsystems, we assign their lower reliability index, because the more complex these subsystems are, the more difficult they will be to achieve high reliability, and therefore the cost will be higher.

For immature subsystem technology, we assign their lower reliability index, which places high reliability requirements on such subsystems that potentially leading to increased development time and increased development costs. 
For sub-systems that operate in harsh environments, we will allocate their higher reliability index, because for these components, harsh environments may increase their failure rate. So they are assigned a high reliability index to ensure that the system can work properly.

For subsystems that are easy to maintain and repair, we will allocate their lower reliability index, because these subsystems are easy to repair even if the failure is also easy to maintain, so the system's reliability can be guaranteed.

For subsystems with high potential for improvement, we assign their higher reliability index, because it is easier to improve their reliability than other subsystems.

For subsystems of high importance, we will allocate their higher reliability index, because the failure of these subsystems, they may affect personal safety and operability.

In addition, the allocation should be combined with the actual situation, and consider other factors, such as the poor reliability of the subsystem and the distribution of high reliability indicators to achieve better overall performance and so on. Reliability allocation is no longer in the subsystem of the existing reliability index or the use of a mature subsystem. At the same time, in the reliability allocation, the reliability index of these subsystems should be removed from the total indicators.

The common reliability distribution methods of $\mathrm{NC}$ lathe are $^{[3]}$ : Equal distribution method, relative failure rate and relative failure probability method, Lagrange multiplier method, dynamic programming method, AGREE distribution method and Arlins distribution method. No matter what kind of distribution method is adopted, the final use of the formula 1 to carry out the distribution of the results of the test:

$$
f\left(R_{1}^{*}, R_{2}^{*}, \ldots R_{i}^{*}, \ldots R_{n}^{*}\right) \geq R_{s}^{*}
$$

$\mathrm{R}_{\mathrm{i}}^{*}$ The reliability index assigned to the i subsystems (or components);

$\mathrm{R}_{\mathrm{s}}^{*}$ Reliability requirements for system requirements.

\section{Mathematical Model of Reliability Distribution in CNC Lathe System}

The reliability distribution of the $\mathrm{CNC}$ lathe system is subject to the Weibull distribution, and the failure rate stabilizes after the run-in period (about 750 to 900 hours). From ${ }^{[7]}$ we can see that the Weibull distribution can describe the stages of the bathtub failure rate curve comprehensively. The reliability function $R(t)$ is computed as indicated in (2)

$$
R(\mathrm{t})= \begin{cases}\mathrm{e}^{-\left(\frac{t-\delta}{\eta}\right)^{m}} & (\delta \leq t ; m ; \eta>0) \\ 1 & (\delta>\mathrm{t} ; m ; \eta>0)\end{cases}
$$

The failure rate function $\lambda(\mathrm{t})$ is computed as indicated in (3)

$$
\lambda(t)=\frac{m}{\eta}\left(\frac{t-\delta}{\eta}\right)^{m-1} \quad(\mathrm{t} \geq \delta ; \mathrm{m} ; \eta>0)
$$

During the above formulas:

$$
\begin{aligned}
& \mathrm{m}-\text { shape parameter } \\
& \delta \_ \text {position parameter; } \\
& \eta — \text { scale parameter. }
\end{aligned}
$$

In the reliability analysis, the failure rate is a constant when $\mathrm{m}=1$, which describes the process of random failure. When we design the reliability of $\mathrm{CNC}$ lathe, it is necessary to consider the random failure process after the running-in period of $\mathrm{CNC}$ lathe. So we can take $\mathrm{m}=1$ into the formula (3) . when $\mathrm{m}=1$, and the corresponding failure rate is $\lambda(t)=1 / \eta$

In addition, in the situation of $m$ and $\eta$ is unchanged, $\delta$ takes a different value, which only the abscissa of the curve is translated without affecting the shape of the curve. In this case, substituting $m=1, \lambda(\mathrm{t})=1 / \eta$ And $\delta=0$ into formula (2),we can compute as indicated in (4).

$$
R(\mathrm{t})= \begin{cases}\mathrm{e}^{-\lambda \mathrm{t}} & \mathrm{t} \geqslant 0 \\ 1 & \mathrm{t}<0\end{cases}
$$

From formula (4), we can see that the system obeys exponential distribution when considering the random failure process of CNC lathe. Moreover, the actual statistical analysis of fitting data is obtained: In most cases, the entire mechanical engineering machinery failure distribution can be considered by the exponential distribution.

The Arlins distribution method ${ }^{[7]}$ is an allocation method that considers the importance degree. The system allows the probability of failure of the system allocated based on the relative proportion of each subsystem to the failure probability of the scheduled time. It is characterized by the existing failure rate of each subsystem based on the failure rate (or failure rate) constant and obey the exponential distribution of the system. The distribution of the 
first-order reliability in the mechanical system mostly uses the failure rate allocation method, and the calculation is simpler and more convenient. So CNC lathes can be applied to the method of reliability of the distribution calculation. The specific steps of reliability allocation are as follows:

(1) According to the accumulated or observed and estimated data, the failure rate of unit (or subsystem) $\lambda_{\mathrm{i}}$ is as follows:

$$
\lambda_{i}=\frac{1}{M_{r}}
$$

During the above formulas:

$\mathrm{M}_{\mathrm{r}}$ the average working time of each component unit or subsystem.

(2) According to the pre-distribution system failure rate $\lambda_{\mathrm{s}}$, the importance distribution factor (also known as the weighting factor) of each unit (or subsystem) $\mathrm{W}_{\mathrm{i}}$ is as follows:

$$
W_{i}=\frac{\lambda_{i}}{\lambda_{s}}=\frac{\lambda_{i}}{\sum_{i=1}^{n} \lambda_{i}}
$$

During the above formulas:

$\mathrm{n}$ - the number of components or subsystems.

(3) Calculate the reliability of the distribution unit (or subsystem) $\mathrm{R}_{\mathrm{i}}$ :

During the above formulas:

$$
R_{i}=R_{S}^{* \mathrm{~W}_{\mathrm{i}}}
$$

$\mathrm{R}_{\mathrm{S}}{ }^{*}$ - the reliability required by the system.

(4) Verify the allocation results.

\section{Reliability Allocation of Various Sub - systems of CNC Lathe}

Through the mathematical model of mechanical systems, we can conclude that each machine is a series between the various subsystems model. In this paper, the Arins distribution method is used to assign the reliability of the system. The CNC lathe system consists of a series system composed of tool post system, clamping system, main drive system and spindle assembly, feed system, chip removal system, power supply and electrical system, CNC system, hydraulic system, cooling system and lubrication system.

According to the literature ${ }^{[5]}$, the mean time to failure (MFET) of each subsystem of the CNC lathe HTC2050 is listed in Table 1.

Table 1 Mean time to failure of each subsystem of HTC2050 CNC lathe



Based on CNC lathe mean time between failure (MTBF) time of China's industrial status and some well-known brands in the United States and Japan is about 500-800 hours, the average mean time to failure of the $\mathrm{NC}$ lathe is 550 hours. In engineering machinery products, the allowable reliability range is $0.90 \sim 0.99^{[7]}$, and considering the reliability level of machinery in China, we set $\mathrm{R}_{\mathrm{s}}{ }^{*}$ to 0.9 . The reliability distribution of $\mathrm{NC}$ lathe system obeys Weibull distribution. The initial run-in period (about 750 to 900 hours), the failure rate tends to be stable and subject to exponential distribution.

(1) According to the average failure time of each subsystem to find the failure rate $\lambda_{\mathrm{i}}$, We can get the following formula.

$$
\lambda_{1}=\frac{1}{M_{1}} \quad \lambda_{2}=\lambda_{4}=\frac{1}{M_{2}} \quad \lambda_{3}=\frac{1}{M_{3}} \quad \lambda_{5}=\frac{1}{M_{5}}
$$

System Failure Rate is $\lambda_{\mathrm{s}}=\sum_{\mathrm{i}=1}^{5} \lambda_{\mathrm{i}}=2.00014 \times 10^{-3} / \mathrm{h}$

(2) The importance distribution factor $\mathrm{W}_{\mathrm{i}}$ of each subsystem is calculated, We can get the following formula. 


$$
\begin{array}{lllll}
W_{1}=\frac{\lambda_{1}}{\lambda_{s}} & W_{2}=\frac{\lambda_{2}}{\lambda_{s}} & W_{3}=\frac{\lambda_{3}}{\lambda_{s}} & W_{4}=\frac{\lambda_{4}}{\lambda_{s}} & W_{5}=\frac{\lambda_{5}}{\lambda_{s}} \\
W_{6}=\frac{\lambda_{6}}{\lambda_{s}} & W_{7}=\frac{\lambda_{7}}{\lambda_{s}} & W_{8}=\frac{\lambda_{8}}{\lambda_{s}} & W_{9}=\frac{\lambda_{9}}{\lambda_{s}} & W_{10}=\frac{\lambda_{10}}{\lambda_{s}}
\end{array}
$$

(3) The reliability Ri of each subsystem is calculated, We can get the following formula.
$R_{1}=R_{S}^{* \mathrm{~W}_{1}}$
$R_{2}=R_{S}^{*} \mathrm{~W}_{2}$
$R_{3}=R_{S}^{*} \mathrm{~W}_{3}$
$R_{4}=R_{S}^{* \mathrm{~W}_{4}}$

\begin{tabular}{|c|c|c|c|c|}
\hline Subsystems & $\mathrm{M}_{\mathrm{i}}(\mathrm{h})$ & $\lambda_{i}\left(10^{-4} / h\right)$ & $\mathrm{W}_{\mathrm{i}}$ & $\mathrm{R}_{\mathrm{i}}$ \\
\hline Turret system & 3848 & 2.5988 & 0.1299 & 0.9864 \\
\hline Clamping system & 4409 & 2.2681 & 0.1134 & 0.9881 \\
\hline $\begin{array}{l}\text { Main drive system and spindle } \\
\text { assembly }\end{array}$ & 5015 & 1.994 & 0.0996 & 0.9897 \\
\hline Feed system & 4709 & 2.1236 & 0.1062 & 0.9888 \\
\hline Chip removal system & 5113 & 1.9558 & 0.0978 & 0.9897 \\
\hline Power and Electrical Systems & 4992 & 2.0032 & 0.0999 & 0.98953 \\
\hline CNC system & 5013 & 1.9948 & 0.0997 & 0.98955 \\
\hline Hydraulic system & 6451 & 1.5501 & 0.0775 & 0.9919 \\
\hline cooling system & 5608 & 1.7832 & 0.0891 & 0.9907 \\
\hline Lubrication system & 5781 & 1.7298 & 0.0869 & 0.9909 \\
\hline
\end{tabular}
$R_{5}=R_{S}^{* \mathrm{~W}_{5}}$
$R_{6}=R_{S}^{*} \mathrm{~W}_{6}$
$R_{7}=R_{S}^{* \mathrm{~W}_{7}}$
$R_{8}=R_{S}^{*} \mathrm{~W}_{8}$
$R_{9}=R_{S}^{*} \mathrm{~W}_{9}$
$R_{10}=R_{S}^{*} \mathrm{~W}_{10}$

(4) Check the allocation result, we can get Reliability $R_{S}=\prod_{i=1}^{10} R_{i}>R_{S}^{*}$

The results are shown in Table 2

Table 2 Calculation result

According to the results, distributing system reliability by using the reliability prediction of failure rate distribution method make the reliability values of each subsystem of CNC lathe are in the allowable range. It is further verified that the reliability distribution of the mechanical system is reasonable. After the reliability distribution of the numerical control lathe, the reliability of each subsystem is obtained. Too high reliability level of each subsystem is not suitable, The cost of promoting each level is a geometric progression. Taking into account the current level of development of the series of CNC lathe is still in the early stages of design, reliability distribution can only be carried out to the subsystems and components. Along with the development of the numerical control lathe and the accumulation of the related reliability data, the reliability of each subsystem can be assigned to each part.

\section{Conclusion}

Understanding and mastering the reliability characteristics of mechanical systems can provide an important basis for manufacturers to develop and optimize mechanical systems and their corresponding combinations. But the domestic status quo of $\mathrm{CNC}$ lathes, manufacturers rarely research and analyze of $\mathrm{CNC}$ lathe reliability. The reason is that $\mathrm{CNC}$ lathes are often large, complex systems with few test samples and fault data. So it is difficult to quantify and calculate accurately. In this paper, the reliability distribution of NC lathe is studied by using the Arling distribution method. Through the reliability test of the whole machine and parts, we can obtain the relevant reliability data. Based on the results of reliability allocation, we can optimize the component design to improve the reliability of the main frame in order to continuously improve and improve product quality. In the rapid 
development of automation technology today, it will provide a reference for the promotion of reliability and basis, so achieving advanced reliability management and improving the mechanical performance are necessary.

\section{Acknowledgment}

The paper is supported by Key Problem Tackling Project of Shaanxi Scientific and Technological Office (2016GY-024).

\section{7.references}

[1] Fan Shaohua. Reliability Allocation of NC lathe Based on the fuzzy theory [D]. Jilin University, 2011: P7

[2] Wang Xin, Lu Chang song. Research on Failure Analysis and Reliability Enhancement Measurement of CNC Lathe [J]. Machine Tool and Hydraulics, 2008: P165

[3] Xiao Jun. Reliability Technology Analysis And Research of CNC Machine Tools [D]. School of Mechanical Engineering Shanghai Jiaotong University, 2007:P51

[4] Yang Pu Liang. An Analysis on the reliability of CNC lathe [D]. Changchun University of Science and Technology, 2013:P640

[5] Xiao Jun, Chen Zhijun, Lu Zhizheng, Guan He, Yang Jianguo. Reliability fuzzy allocation of CNC machine tools [A]. School of Mechanical Engineering Shanghai Jiao Tong University,2006:P54

[6] Yang Pu-liang, LI Yu-ju. An Analysis on the reliability of CNC lathe [J]. Journal of Changchun University, 2012:P638

[7] Liu Pin, Reliability Engineering Foundation [M], Beijing: China Metrology Press, 2002

[8] He Ming-li, Xiang Xiao-dong, Guo Yin-liang. New approach on system reliability distribution by component importance in FTA [J]. Safety and Environmental Engineering, 2009:P63

[9] Yang Zhao-jun, Chen Chuan-hai, Chen Fei, Li Guo-fa. Progress in the Research of Reliability Technology of Machine Tools[J]. Journal of mechanical engineering, 2013:P134 